\title{
Revisión de literatura sobre educación contable en Colombia ${ }^{1}$
}

\author{
Ruth Alejandra Patiño Jacinto ${ }^{2}$ \\ Gloria Milena Valero Zapata ${ }^{3}$ \\ Martha Giovanna Acosta Sahamuel ${ }^{4}$ \\ José Fabián Parra Acosta ${ }^{5}$ \\ Jairo Alonso Bautista
}

1 Artículo de revisión derivado de la investigación denominada "Caracterización de los currículos de contaduría pública en programas profesionales de universidades colombianas", desarrollada en la Facultad de Contaduría Pública de la Universidad Santo Tomás sede Bogotá y financiado por la Asociación Colombiana de Facultades de Contaduría Pública [ASFACOP, 2014].

2 Contadora pública, Universidad Nacional de Colombia. Magíster en Ciencias Económicas, Universidad Santo Tomás. Coordinadora del Centro de Investigaciones de la Facultad de Contaduría Pública de la Universidad Santo Tomás, Bogotá, Colombia. Miembro del Grupo de Investigación “Contaduría, Información, Control e Impacto Social”. Líneas de desarrollo: Educación contable, Normas internacionales de información financiera, Investigación contable, Hacienda pública. Correo electrónico: alejandrapatino@usantotomas.edu.co.

3 Contadora pública y magíster en Medio Ambiente y Desarrollo, Universidad Nacional de Colombia. Docente de la Facultad de Contaduría Pública de la Universidad Santo Tomás, Bogotá, Colombia. Miembro del Grupo de Investigación “Contaduría, Información, Control e Impacto Social". Líneas de desarrollo: Educación contable, Investigación contable, Contabilidad ambiental. Correo electrónico: gloriavalero@usantotomas.edu.co.

4 Contadora pública, Universidad Nacional de Colombia. Magíster en Educación, Universidad Santo Tomás. Docente de tiempo completo, Universidad Militar Nueva Granada, Facultad de Ciencias Económicas Campus, Programa de Contaduría Pública, Cajicá, Colombia. Correo electrónico: martha.acosta@unimilitar.edu.co

5 Docente de la Universidad Santo Tomás y de Universidad Nacional de Colombia. Director del Grupo de Estudios en Contabilidad de Costos y Control de Gestión -Control+Ge de la Universidad Nacional de Colombia. Correos electrónicos: jose.parra@usantotomas.edu.co,jfparraac@unal.edu.co

6 Contador público, Universidad Nacional de Colombia. Magíster en Administración Pública, Escuela Superior de Administración Pública. Docente 
Patiño, R., Valero, G., Acosta, M., Parra. J. \& Bautista, J. (2016). Revisión de literatura sobre educación contable en Colombia. Activos, 26, 61-99.

\section{Clasificación JEL: M40}

\section{Resumen}

En el presente trabajo se realizó la revisión de publicaciones desarrolladas en el contexto colombiano sobre educación superior contable, con el propósito de caracterizarlas y analizarlas. Para ello, los documentos identificados se clasificaron en las siguientes categorías: plan de estudios, desempeño y perspectiva profesional, metodología enseñanza-aprendizaje, docencia universitaria, productividad académica contable, ética, historia contable, calidad y, finalmente, aspectos generales sobre educación y la investigación. La metodología utilizada fue cualitativa-documental, por cuanto se basó en la revisión de publicaciones en el tema en mención. Entre los principales hallazgos se encontró que la mayoría de los trabajos gira en torno a temas tradicionales, como plan de estudios y ética, mientras que los avances en historia de la educación contable aún son escasos.

\section{Palabras clave}

Educación contable, currículo contable, plan de estudios, investigación contable, publicaciones contables.

investigador de la Universidad Santo Tomás. Grupo de investigación Contaduría: Control y Gestión de la Información en las Organizaciones. Correo electrónico: jairobautista@usantotomas.edu.co 
Patiño, R., Valero, G., Acosta, M., Parra. J. \& Bautista, J. (2016). Review of literature on accounting education in Colombia. Activos, 26, 61-99.

\begin{abstract}
In this paper, it is presented a review for characterizing and analyzing publications about countable college education. This research was developed in the Colombian context. In order to do this review, the material identified was classified in the following categories: curriculum, performance and professional perspective, teaching-learning methodology, university teaching, countable academic productivity, ethics, accounting history, quality and, finally, general aspects about education and research. The methodology utilized was qualitative - documentary, because it was based on the review of publications on the subject in question. Among the key findings, it was found that most researches treat around traditional topics as curriculum and ethics, while issues as advances in the history of accounting education are still scarce.
\end{abstract}

\title{
Keywords
}

Accounting education, curriculum of accountancy, curriculum, accounting research, accounting publications.

Patiño, R., Valero, G., Acosta, M., Parra. J. \& Bautista, J. (2016). Révision de la littérature sur léducation comptable en Colombie. Activos, 26, 61-99.

\section{Résumé}

Dans cet article, on a réalisé la révision des publications élaborées dans le contexte colombien au sujet de l'enseignement supérieur de la comptabilité, dans le but de les caractériser et de les analyser. Pour ce faire, les documents identifiés ont été classés dans les catégories suivantes: programme deétudes, 
performance et perspective professionnelle, méthodologie enseignementapprentissage, enseignement universitaire, productivité académique comptable, éthique, histoire comptable, qualité, et finalement aspects généraux sur léducation et la recherche.

La méthodologie utilisée fut qualitative-documentaire, parce qu'elle s'est fondée sur la révision des publications sur le sujet en question. Parmi les principales conclusions, on a constaté que la plupart des travaux tournent autour de thèmes traditionnels, tels que les programmes détudes et l'éthique, alors que les progrès dans l'histoire de l'éducation comptable sont encore rares.

\section{Mots clés}

Éducation comptable, programme comptable, programme d'études, recherche comptable, publications comptables.

\section{Introducción}

Durante los últimos años, se han presentado a la comunidad contable valiosos aportes teóricos en torno a la educación contable y las distintas categorías que la constituyen; sin embargo, como un campo específico, el currículo es un concepto que debe ser revisado y analizado. De esta manera, el grupo de investigación realizó un trabajo de corte documental en el que se recopiló, revisó, sistematizó y analizó cada uno de los documentos encontrados en las siguientes publicaciones: Revista Lúmina, Revista Contaduría de la Universidad de Antioquia, Revista Cuadernos de Contabilidad, Memorias del I, II y III Encuentro Nacional de Profesores de Contaduría Pública, así como de distintos libros que reflexionan en torno al concepto de currículo en programas de Contaduría Pública. El criterio inicial de la revisión documental se centró en las revistas indexadas; no obstante, posteriormente se encontraron trabajos importantes en el tema de educación contable en Colombia en libros y en el encuentro de profesores 
realizado cada dos años; adicionalmente, aunque la Revista Contaduría en este momento no se encuentra indexada, es un referente muy importante por ser la de mayor trayectoria en el país.

A partir de esta vasta revisión documental, y previamente discutidas las categorías de análisis (plan de estudios, desempeño y perspectiva profesional, metodología enseñanza-aprendizaje, docencia universitaria, productividad académica contable, ética, historia contable, calidad y, finalmente, aspectos generales sobre educación e investigación), las cuales son el producto de una revisión previa utilizada por Valero y Patiño (2010) en journals internacionales, se evidencia la posición de los distintos autores en cada categoría y su principal aporte en la construcción del concepto de currículo para la contaduría pública.

El principal interés del grupo de investigación se centró en la comprensión de la postura teórica de la comunidad contable en torno al currículo y a los referentes que lo constituyen, además de realizar un inventario de las publicaciones del tema en cuestión, con el fin de evidenciar las principales discusiones teóricas o la falta de estas, estudio necesario y pertinente para la disciplina y la profesión contable.

En cuanto a la metodología desarrollada, esta se realizó por etapas. La primera se centró en la definición de las fuentes que se iban a usar, la razón para incluirlas y el rango de tiempo que se revisarían; inicialmente se definió como período de revisión los últimos 30 años, pero conviene advertir que dicho término se fue ampliando a partir de los documentos que se fueron encontrando, de tal forma que finalmente el rango analizado fue de 1987 a 2013. Con posterioridad, y una vez recopilados cada uno de los documentos en medio electrónico o físico, se procedió a sistematizar la información con la ayuda de una matriz en la que se consolidaron los documentos y se organizaron por fuente, autor y año, para luego iniciar el proceso de lectura y análisis de los aportes teóricos y su clasificación en cada una las categorías predefinidas al inicio, de tal manera que se describieran las posiciones de los distintos autores respecto a cada categoría y se evidenciara el estado teórico del objeto de estudio: el currículo en contaduría pública. 
Entre los resultados generales de la caracterización, es de resaltar el impacto de los encuentros de docentes en el significativo incremento de la producción de documentos relacionados con el tema objeto de revisión, lo cual es normal por la naturaleza del evento y se contrasta en la tabla 2: Composición de documentos revisados por fuente, así como en la tabla 3 : Distribución por categoría de análisis en el tiempo, en el que los períodos con mayor productividad tienen directa relación con el desarrollo de los encuentros. De igual forma, se logran identificar los autores colombianos con mayor productividad por categoría, aspecto que permite reconocer y resaltar el trabajo que se viene desarrollando en las diferentes regiones del país en respuesta a las necesidades de cada contexto en particular.

\section{Resultados}

El currículo es un referente obligado en las instituciones de enseñanza porque es el lugar donde se materializan las políticas educativas y se plasman las intencionalidades por medio de la práctica docente; se hacen apuestas educativas que propenden hacia la calidad de la educación y se presenta como un componente dinámico en la actualidad. El currículo, como campo de conocimiento, tuvo sus inicios en los Estados Unidos y deviene del latín que significa carril o senda. Para Bolívar (2008, p. 12):

El curriculum puede ser entendido como una cultura, como un conjunto de normas, pautas, valores que en un determinado tiempo dominan los discursos políticos y las prácticas docentes, se ha configurado como una plataforma conceptual para analizar, deliberar y consensuar cuál es y debe ser la educación ofrecida, y los medios y formas a emplear para lograrlo. Como cuerpo teórico de reflexión ofrece, en efecto, un campo para argumentar, decidir y planificar cuál deba ser la educación deseable. El ámbito de estudio del curriculum posibilita pensar y desvelar los contextos sociales, políticos e ideológicos que subyacen en las propuestas curriculares y en las prácticas educativas.

Barragán (2009, p. 18) planteó que los currículos en contaduría presentan un enfoque muy particular llamado "enfoque tecnocrático", en el que 
el Estado, por medio de las normas, es la figura que orienta la educación a la satisfacción de necesidades del sector productivo, independiente de las necesidades sociales, culturales y económicas, y se dirige a capacitar mano de obra calificada y a satisfacer al sector productivo.

A continuación, se señalan las principales discusiones teóricas en torno al currículo en las siguientes categorías definidas previamente.

\section{Plan de estudios}

El currículo puede considerarse como una forma de organizar administrativamente la enseñanza por medio de un plan de contenidos:

Es solo un componente dentro del currículo, ya que debe estar acompañado de la definición del perfil del estudiante, la misión y visión del programa, las características en materia de investigación, entre otros, con el fin de propender [hacia] calidad en el respectivo programa. (Valero y Patiño, 2010).

Además, contempla una serie de documentos que contienen un conjunto de componentes interrelacionados (objetivos, contenidos, metodología, actividades, recursos y previsiones de evaluación) denominados programas o syllabus, los cuales posibilitan el cumplimiento de las principales finalidades de formación planteadas por una institución educativa.

De acuerdo con la revisión bibliográfica y en relación con los planes de estudio de Contaduría Pública, las principales críticas radican en su composición fragmentaria y asignaturista, así como por "la ausencia de núcleos de integración o proyectos que permitan poner en congruencia los diversos saberes en los que se forma al estudiante" (Ospina, 2009, p. 7), situación que se hizo evidente en 1996 con la propuesta de renovación curricular para el país, liderada por el C. P. Dagoberto Pinilla, la Fundación para la Investigación y Desarrollo de la Ciencia Contable [FIDESC] y el Instituto Colombiano para el Fomento de la Educación Superior [ICFES]. La nueva propuesta estructuró el plan de estudios en los ciclos disciplinario, 
profesional, de proyección e innovación y de opción de grado, con una duración de cinco años; en este proyecto se identificaron las siguientes novedades: la opción de seminario como estrategia para desarrollar la creatividad en el ciclo de proyección e innovación; la necesidad de la fundamentación disciplinaria durante el proceso de formación; la importancia para la profesión de vincular conocimiento de otras disciplinas, como lo son la filosofía, la antropología y la sociología; y, por último, la opción de grado como posibilitadora social de los intereses de los estudiantes (Zapata, 1997, p. 68). Sin embargo, a pesar de ser innovadora en su momento histórico al pensar en la profesión no solo en un plano técnico-práctico e incluir discusiones en asuntos de educación dentro de la comunidad contable, la propuesta final mantuvo el mismo corte asignaturista ya criticado por esa época y no logró el impacto esperado en las reformas curriculares de las distintas facultades de Contaduría Pública del país.

En el diseño y rediseño curricular por lo general se trabaja en el plan de estudios, y es uno de los ejes temáticos de mayor reflexión en la educación contable en Colombia, por un lado, en la búsqueda de currículos que tengan pertinencia social y, por el otro, para cumplir con las exigencias normativas. Es por ello que Zapata (1998, p. 80) sugería que las facultades de Contaduría crearan "grupos de discusión sobre los problemas y las propuestas de interés común", en los que compartieran los avances y retrocesos de los procesos encaminados en esa exploración. En la misma línea, Ariza y Soler (2004, p. 192) plantean que las facultades de Contaduría Pública deberán generar reformas curriculares que cumplan con los requerimientos del Estado, pero fundamentalmente "que vayan más allá de las respuestas formales y coyunturales"; para ello estructuran unos lineamientos por seguir para la reforma en la investigación contable.

Un ejemplo de estas actualizaciones se encuentra en el plan de estudios del programa de Contaduría Pública de la Pontificia Universidad Javeriana frente a los retos que generó la Ley 1314 de 2009, enfocándose en el cumplimiento de una formación en estándares internacionales de contabilidad, proceso que iniciaron tempranamente para hacer frente a la nueva realidad contable del país (Valderrama, Castro, Arias, Bejarano, Garzón, Salgado, 
Rueda, Espinosa y Sosa, 2009). En la misma dirección del proceso de convergencia a normas internacionales, se establece como un imperativo vincular al plan de estudios asignaturas relacionadas con ciencias políticas, negocios internacionales, manteniendo su complementariedad con economía, de forma que un estudiante de Contaduría Pública pueda comprender e interiorizar "el origen y el alcance de la contabilidad internacional y sus implicaciones en las sociedades actuales y particularmente para Colombia" (Rueda-Delgado, 2013, p. 27).

A partir de las múltiples exigencias que el contexto le plantea a los programas de Contaduría Pública, se pueden resaltar nuevos requerimientos asociados con la diversidad cultural del país y con la denominada contabilidad cultural, centrada en la relación de la contabilidad y la cultura, aspectos que terminan incidiendo en el currículo contable y que plantean la necesidad de un currículo que "incorpore a su manera, los principios, los valores, los derechos y responsabilidades de los individuos y las comunidades que conviven en la geografía nacional" (Giraldo, 2013, p. 17). Es decir, se trata de una formación contable que contribuya con la inclusión y el reconocimiento de "los grupos étnicos y culturales colombianos", no solo desde la esfera financiera, sino desde la simbología implícita en la idiosincrasia de los pueblos; sin embargo, no es claro cómo hacerlo posible y presupone preocupaciones distintas a las convencionales.

De la mano de la propuesta anterior, se encuentra la vinculación de las humanidades en la formación contable como un elemento que podría garantizar la tan anhelada formación integral, sin descuidar el componente profesional y justificando su vinculación en los programas de Contaduría Pública por razones como su contribución a:

Primero, descifrar y proyectar caminos de libertad para pensar los diversos problemas colombianos que facilitan o imposibilitan el desarrollo de la disciplina y del quehacer contable; segundo, porque facilitan poner en duda los pensamientos y las ideas que subyacen al conocimiento profesional desarrollado y amparado por las presiones de los usuarios de la información y de gremios profesionales que no han problematizado las 
teorías y los modelos que se utilizan para producir, procesar e interpretar la información contable financiera. (Rojas y Giraldo, 2013, p. 9).

El enfoque de un nuevo modelo curricular, según los estudios identificados, tiene que comprometer cuatro temas centrales: (i) el proceso de formación, (ii) los conocimientos, (iii) las destrezas y (iv) la orientación profesional. A su vez, se resalta cómo la experiencia de formación en Contaduría Pública de las universidades colombianas, en una gran mayoría de los casos, demuestra que ha respondido con eficiencia a la formación técnica más que investigativa y práctica, más que teórica y conceptual, pero en particular, altamente desconectada de la realidad, en la medida en que su núcleo es la aplicación e interpretación regulativa (Vásquez, 2002).

Frente a los planes de estudios, Patiño y Santos (2009) plantean las siguientes condiciones de actualidad para la carrera de Contaduría Pública en Colombia: los programas curriculares toman las recomendaciones dadas por los organismos internacionales, los cuales no presentan grandes diferencias entre sí (uno de los referentes es IFAC ${ }^{7}$ ); no obstante, se encuentran elementos distintivos del país, los cuales han permitido insertar algunos cambios y trascender lo instrumental por medio de la "interdisciplinariedad, la ética y la cultura", además de la "flexibilidad e investigación"; adicionalmente, se encuentra una gran heterogeneidad entre las asignaturas de los diferentes programas académicos.

Es pertinente resaltar que para Gracia (2011), la investigación se debería integrar al currículo con el fin de generar una "masa crítica" y un carácter investigativo en los estudiantes, lo cual, de la misma forma, contribuiría a la formación integral, aspecto que se ha convertido en preocupación en la educación actual.

Finalmente, uno de los trabajos de mayor relevancia es el de RuedaDelgado (2009a), quien, en el marco de los desarrollos del convenio académico interfacultades, que reúne 14 programas de Contaduría Pública del

$7 \quad$ Federación Internacional de Contadores. 
país, presenta una comparación de los contenidos currículares de dichos programas, resaltando sus filosofías, los componentes de sus planes de estudios y las orientaciones profesionales. Este documento permite señalar algunas tendencias analíticas, por ejemplo, las isometrías de los programas en términos de los contenidos de los planes de estudios y las vocaciones y capacidades profesionales que despliegan.

\section{Desempeño y perspectiva profesional}

Conocer las demandas que la sociedad actual hace del contador público propicia el escenario necesario para que las IES dirijan el proceso de formación de tal manera que el profesional recién egresado sea útil y pertinente para el contexto; es por ello que Zapata (1997, p. 64) presentó el perfil profesional del contador público para el siglo XXI desde un enfoque integral, el cual debe contar con características como la biofilia o amor a la vida, solidaridad, vocación, democracia y espíritu dialógico. Zapata afirma que la relación maestro-alumno debe basarse en la confianza y destaca la necesidad de que los contadores adquieran un pensamiento global e incorporen en su ejercicio profesional un estilo gerencial y de liderazgo.

Frente a esta categoría, las preocupaciones son diferentes dependiendo de la mirada del actor desde el cual se analiza; sin embargo, la principal propuesta está encaminada a potenciar la pertinencia de la formación académica en el entorno y se centra en la conexión entre

la universidad, el mercado laboral y el Estado, donde el programa académico se retroalimente de los requerimientos del contexto y diseñe estructuras curriculares que permitan, mediante el desarrollo de competencias, una adecuada formación personal y profesional. (Revelo y Bennett, 2013, p. 1).

\section{Metodologías de enseñanza-aprendizaje}

En esta categoría, los trabajos encontrados son diversos y exploran diferentes facetas de esta función sustantiva de las universidades a partir del 
análisis de modelos, metodologías pedagógicas, estrategias, instrumentos y didácticas, entre otros.

Por ejemplo, en el trabajo de Cárdenas-Londoño (2009), Papel de las metodologías de enseñanza del proceso lectoescritural en la educación superior, el autor logra hacer recomendaciones frente a la actividad docente en Contaduría Pública, y entre los diferentes aspectos que vincula se centra en una serie de registros que los docentes deberían seguir, tales como:

Intente redactar su clase, lleve una bitácora didáctica o un diario (...) Para fomentar la escritura en el aula: pida siempre a sus estudiantes la construcción y lectura pública de uno o dos párrafos que sinteticen los aspectos centrales de cada clase y sus aprendizajes. Evalúe su escritura y la de sus estudiantes. (Cárdenas-Londoño, 2009, p. 98).

Otros trabajos analizan el papel de las tecnologías de información y comunicación [TIC] en la formación de contadores públicos como un aspecto no muy explorado; algunos enfatizan en cómo se configuran las TIC en un factor potenciador de las capacidades de los estudiantes para aplicar el conocimiento. Pero se requieren esfuerzos gubernamentales, institucionales e individuales que incrementen las motivaciones para generar cambios significativos en los procesos de enseñanza-aprendizaje mediados con las TIC para convertir los escenarios de formación en espacios donde los estudiantes apliquen en la práctica lo que están aprendiendo (Barreto et al., 2011).

Por otra parte, una estrategia pedagógica que se planeta para dar respuesta a las exigencias de las nuevas generaciones de estudiantes de Contaduría consiste en "las aulas", entendidas como "espacios académicos de reflexión, discusión y análisis de los temas desarrollados en vínculo con la realidad a través del trabajo colectivo organizado" (Moreno, 2006, p. 53), sujeto a características mínimas del grupo y a una evaluación diagnóstica permanente. Del mismo modo, se encuentran propuestas centradas en la "enseñanza problémica", que permiten innovar el trabajo en el aula mediante la generación de situaciones de incertidumbre y contradicción que potencien la reflexión disciplinaria en contexto (Díaz, 2006). 
Roncancio y Camargo (2013) exploran el papel y la incidencia del "libro de texto" en la educación contable en Colombia, así como algunos de los criterios que los docentes utilizan para su selección, resaltando lo tradicional del modelo de enseñanza-aprendizaje en contabilidad, junto con la importante utilización de los libros de texto frente a otras opciones pedagógicas.

De otro lado, es claro que una variable poco estudiada en educación contable tiene que ver con la didáctica; frente a lo anterior Martínez (2011, p. 140) plantea que "hace falta resignificar el acto educativo desde una didáctica medida por la creatividad que recree el pensamiento científico y vivencie la experiencia de búsqueda de conocimiento".

Con base en lo anterior, Barragán $(2009$, p. 6) señala que se presentan dos posiciones frente a la orientación que debe tener el proceso de formación. En primer lugar, los que señalan la importancia de enfocar la formación hacia la "práctica", es decir, desarrollar habilidades para el mercado laboral, formar "buenos empleados". Esta tendencia la denomina activismo, enfocar el proceso de formación y el curriculum a satisfacer necesidades del momento y de las distintas organizaciones, sin tener referentes conceptuales sólidos e indagar por las necesidades económicas y sociales. En segundo lugar, están los verbalistas, que se enfocan en la formación en discursos y contenidos sin relación ninguna con las necesidades sociales, incluso sin relación con la acción educativa y las condiciones reales de las universidades. En ese orden de ideas, el autor propone no formar en una tendencia específica, en la medida en que el enfoque de formación debe ser integral, que considere la persona, la sociedad, las organizaciones, la cultura y el ambiente, y no se debe formar con una orientación netamente teórica o técnica.

Desde una visión alternativa, Sánchez (2011) desarrolla un conjunto de reflexiones sobre los componentes éticos implícitos en la formación del contador público y propone el desarrollo de un conjunto de procesos pedagógicos para el desarrollo de competencias socioafectivas orientadas especialmente a estudiantes de Contaduría. En este sentido, el autor destaca la importancia de esta propuesta en la medida en que: 
En el nivel universitario se espera que el estudiante llegue dotado de los instrumentos afectivos evolutivos propios de su desarrollo cronológico y emocional y que la universidad se encargue de formarle con los propios de su adolescencia y adultez para el ejercicio profesional. La institución educativa debe lograr, [por medio] de estas competencias, impactar o afectar (de afecto y afectación) al dicente para lograr el interés suficiente y necesario, la imprescindible motivación para aprehender, la comprehensión (sic) acerca de la importancia de las disciplinas que nutren la profesión, sobre el papel de la misma y su incidencia en la vida socioeconómica, cimentar un conjunto de actitudes y valores que orienten la forma y el fondo al momento de relacionarse consigo mismo y con los demás, y con el mundo y el conocimiento. (Sánchez, 2011, pp. 26-27).

La obra en referencia contiene un conjunto de reflexiones sobre las dimensiones socioafectivas, basadas en los trabajos del maestro Miguel de Zubiría, y las aplica a las dimensiones de la educación contable, acompañadas de un conjunto de ejercicios y materiales multimediáticos.

En cuanto metodologías de enseñanza-aprendizaje en áreas específicas, se encuentra el trabajo de Villarreal (2011) en auditoría, mediante la propuesta de aprendizajes significativos, por medio de laboratorios, "usos de sistemas de información y aplicativos tecnológicos", todo con el fin de lograr un "papel dinámico" del futuro auditor.

\section{Docencia universitaria}

Trabajos como el denominado Retos de la academia contable enfatizan en que los académicos de la ciencia contable en Colombia se deben preparar para responder de la mejor manera a los retos que el proceso de convergencia contiene. Lo anterior implica un proceso de aprendizaje y de investigación que busque arrojar resultados válidos y aplicables en las condiciones particulares del país (Rodríguez, 2010).

Otros enfatizan en cómo el docente es un agente lleno de discursos propios que le permiten tener una integración con el campo. 
El docente en la cotidianidad se convierte en replicador y/o transformador de las pugnas ideológicas de su campo profesional y académico. Estos docentes han desarrollado síntesis que evocan "voces" de las propuestas sobre los ámbitos disciplinario y académico... (León, 2011).

Cardona (1998) afirma que existen dos posturas antagónicas del maestro al resolver problemas contables junto con sus estudiantes. En la primera está el maestro que plantea varias opciones a un tema sin profundizar en las características y dinámicas de cada solución propuesta, y del otro lado está el docente que expone una solución como única verdad, la cual debe retransmitirse entre los estudiantes. A lo anterior el autor agrega que la adecuada es la postura que logra que el maestro se transforme desde un proyecto educativo y pedagógico, estableciendo las condiciones, los requisitos y las particularidades que le permitan al estudiante desarrollar su propia comprensión del tema. En este apartado se logra identificar la escasez de trabajos sobre el papel de uno de los principales actores de la educación contable.

\section{Productividad académica contable}

Trabajos como "Orígenes de la producción académica contable", presentado en el editorial del volumen 10 de la Revista Cuadernos de Contablidad, enfatizan en que se debe "desplegar una serie de estrategias y propuestas para beneficio de la profesión, la disciplina, la comunidad contable nacional y las revistas científicas contables". Se presentan a continuación algunas de las propuestas, las cuales no son excluyentes entre sí (Rueda-Delgado, 2009b): coordinar con los grupos de investigación de cada institución la generación de productos pertinentes, oportunos, profundos e inéditos, sin que se trate de aumentar la "productividad" o la producción de artículos, como si se tratase de la fabricación de bienes materiales, para potenciar dichos grupos; incentivar la formación de redes de trabajo y la generación de incentivos para producción de nuevos trabajos de investigación.

A partir de lo anterior, se requieren estrategias que garanticen el aprovechamiento de la producción académica, por ejemplo, el uso de artículos en 
las aulas de clase no solo ayudará a visibilizar las revistas ante la academia especializada, sino que contribuirá a la formación integral reflexiva de los futuros contadores públicos, quienes podrán argumentar y construir sus propios pensamientos y discursos (Rueda-Delgado, 2011).

Ejercicios prácticos como el denominado Una aproximación al uso de herramientas de gerencia estratégica de costos en instituciones privadas de educación superior (Cárdenas-Mora, 2011) muestran cómo el desarrollo de la investigación permite profundizar diferentes campos, en este caso en materia de gerencia estratégica y de costos, haciendo la salvedad de que aun cuando se han hecho aproximaciones importantes respecto a las variables analizadas, no se puede decir que el tema se haya agotado. A su vez, el ejercicio en mención rescata la trascendencia que tiene la educación superior en el país y las múltiples consideraciones que quedan por hacer y, por lo mismo, da lugar a otros trabajos. Así, caben otras investigaciones que permitan hacer comparaciones, como la que en materia de gerencia estratégica de costos se está dando en el sector de la educación superior pública (Cárdenas-Londoño, 2011).

\section{Ética}

La ética es una categoría recurrente en la educación contable colombiana, principalmente por los sonados casos de crisis en la confianza en la profesión y, por ende, en los profesionales contables. Respecto a este punto, se encuentran planteamientos que tienen como propósito abordar el tema desde el aula de clase. Entre las diferentes propuestas está la vinculación del "dilema moral como estrategia pedagógica", que pretende enfrentar al estudiante de Contaduría a situaciones concretas que impliquen la elección de como mínimo dos alternativas que deben ser argumentadas y propender hacia el desarrollo del criterio profesional frente a la subjetividad implícita en el quehacer contable: "los dilemas deben cumplir la condición de crear un conflicto cognitivo que rompa la seguridad del razonamiento del estudiante" (Herrera, 2013, p. 12). 
Los grandes escándalos de fraudes financieros alteraron a la comunidad contable a nivel mundial; esta serie de eventos generó que la información contable pasara del ámbito privado a un bien público, situación que amerita una conciencia ética en la mente y en las acciones de los contadores públicos. En palabras de Gonzalo y Garvey (2009, p. 14), se trata de tener un conjunto de profesionales que comprendan el espíritu de las normas contables, las apliquen según su finalidad y sientan la responsabilidad de elaborar o auditar una información financiera útil para cumplir su objetivo social más amplio, lo cual se logra cuando en el aula se discuten contextualmente las normas, se ubican en el momento histórico en que se han gestado, se conocen las consecuencias económicas y se les da un amplio sentido ético y social, y no solamente se dedica el tiempo a revisar el aspecto técnico.

En relación con la ética, el contador público como docente es ético, muestra respeto, tiene valores, tiene una buena actitud y difunde la información reciente (Celaya, López y Aceves, 2008).

Hay otros documentos un poco más especializados, como el artículo titulado "Educación bioética para vivir, convivir y habitar correctamente. Un plus a la capacitación profesionalizante" (Cely-Galindo, 2011), el cual concluye que

(...) las ciencias y tecnologías no son suficientes en los procesos educativos de las actuales y futuras generaciones. Quizás ellas solas podrán llevarnos a formar monstruos humanos, altamente capacitados para destruir y autodestruirse (...). (Cely-Galindo, 2011).

Lo ético comprende, ante todo, las disposiciones del hombre en la vida, su carácter, sus costumbres y, naturalmente, también lo moral. En realidad, se podría traducir por modo o forma de vida en el sentido hondo de la palabra, a diferencia de la simple manera. Así, la ética - vista desde el carácter o modo de ser de la persona - resulta estar cimentada sobre la estructura misma de la persona, y esa estructura de la persona significa su propia habitación, su morada (Zubiri, 1987, p. 248). Entendiendo el concepto desde esta dimensión, Cardona (1997) observó como una manifestación ética del 
profesional contable el reconocer la responsabilidad social de contar con conocimiento y prestar servicios actualizados, y el compromiso social de construirse a sí mismo como mejor profesional, aspectos que se materializan gracias al acceso a la educación permanente, situación ya legislada por los organismos reguladores de la profesión contable en sus códigos de ética.

\section{Historia de la educación}

En este campo de análisis, los trabajos más destacados han hecho aportes fundamentales para la comprensión de los procesos evolutivos de la educación contable en Colombia. El primero de ellos es el trabajo de Cubides (1994), que en una muy amplia explicación reconstruye la historia de la educación contable en Colombia desde sus antecedentes en el siglo XIX hasta los desarrollos alcanzados a finales de la década de los años ochenta del siglo XX. Este trabajo, pionero en los estudios sobre el tema, aborda las relaciones que se tejen entre la enseñanza de la contaduría pública y el contexto institucional y social que se tejía; en este sentido los aportes corresponden a una mirada socio-histórica en la que se referencian los desarrollos de la educación contable en relación con los sistemas políticos, económicos y sociales dominantes en cada época.

Adicionalmente, el trabajo de Cardona y Zapata (2006) contiene aportes fundamentales para la comprensión de los procesos educativos contables en el siglo XIX. Este documento recurre a un riguroso trabajo de archivos en el que los autores logran detallar las regulaciones académicas, los elementos de la docencia, las instituciones que ofertaban estudios sobre teneduría de libros y aritmética comercial, los textos o manuales que circulaban en la época y, finalmente, los contenidos de los programas que ofrecían las instituciones para la enseñanza de la contabilidad.

Aun cuando esta categoría (historia de la educación contable) es un factor relevante cuando de investigación se trata, se evidencia la falta de trabajos desarrollados en ello, lo cual se queda en unos cuantos autores tradicionales, aunque con aportes importantes, sin embargo, pocos frente a otros temas de mayor productividad. 


\section{Investigación}

Un autor que menciona la investigación como elemento fundamental dentro del currículo es Araújo (2000), quien comenta que uno de los elementos fundamentales en el cambio en la educación en los últimos treinta años ha sido el espíritu investigativo, por medio del cual se incentiva a que el estudiante

fomente y auspicie la investigación y el progreso científico y tecnológico de la contabilidad y la contaduría pública; que se relacione interdisciplinariamente; que desarrolle nuevas metodologías y enfoques epistemológicos contables; que pueda plantear correctamente los problemas y abordar sus soluciones. (Araújo, 2000, p. 91).

De igual forma, se resalta la necesidad de formar en investigación con el fin de mejorar el análisis de los problemas y de generar un sentido crítico en los estudiantes de Contaduría Pública. De acuerdo con Ríos (2013, p. 43):

La formación para la investigación es indispensable en el ejercicio de la docencia (...), los acerca a la realidad organizacional y les permite ser parte de la solución de los problemas que se presentan en el contexto.

Conocer el desarrollo del pensamiento contable es vital en el proceso de formación de contadores públicos; este conocimiento propicia en los profesionales una mirada crítica y reflexiva frente a la disciplina y la profesión; enfoca los problemas de la contabilidad en forma lógica, ordenada y coherente; da una explicación de los fenómenos contables orientados más desde el ser que desde el deber ser. Sin embargo, todo este conocimiento tan necesario para el contador público aún no ha gozado de la suficiente atención por parte de los centros universitarios, al centrar las finalidades de enseñanza en términos pragmáticos que respondan a las necesidades del ambiente de los negocios (Zapata, 1997, p. 32). Consolidar en el proceso de formación este tipo de conocimiento aporta de manera sustancial en los procesos de investigación formativa y formal, tan necesarios en las facultades de Contaduría Pública del país, y propicia en los estudiantes y 
docentes un espíritu investigativo que contribuye a desarrollar la disciplina, la profesión y la sociedad.

\section{Calidad}

El concepto de calidad cobra especial interés en la actualidad y ha sido abordado por distintos autores. Al respecto, Cardona y Rivera (2002, p. 73) señalaron que en contaduría pública la calidad se refiere a i) acreditación de programas, ii) certificación de profesionales mediante exámenes, iii) revisión de la calidad mediante pares y iv) estándares mínimos de registro calificado para los programas, procesos de gestión propuestos por los organismos reguladores nacionales e internacionales, el Estado, entidades multilaterales de orden superior y la misma comunidad contable, con el fin de garantizar pertinencia y calidad en los servicios prestados por los profesionales, aumentar el impacto de la profesión en la sociedad y armonizar las prácticas contables globalmente.

De otra parte, Rodríguez (2008, p. 200) señala cómo el concepto de calidad, visto desde la gestión de calidad total [GCT], centrado en los procesos y productos, se ha expandido hacia las instituciones de educación superior desde los años noventa. Entre los argumentos detractores, Rodríguez comenta que considerar la GCT en los procesos educativos se ajusta a los supuestos de la política neoliberal, para el beneficio de unos pocos. Igualmente, Santana $(2009$, p. 8$)$ indica que la GCT se aplica si la enseñanza se entiende como una actividad técnica, pero las organizaciones educativas son realidades sociales construidas y no orientadas al mercado.

En relación con la calidad de la educación superior contable, Gómez (2007) plantea una postura crítica frente a la adopción de estándares para los procesos relacionados con la calidad de la formación de contables, en cabeza de la International Federation of Accountants [IFAC], y resalta como ejes centrales de discusión los medios de enseñanza y los contenidos, así como una serie de problemáticas propias la contabilidad en Colombia. A este propósito: 
La propuesta de IFAC es un referente, hoy, obligado por las prescripciones de la autoridad global, pero no es el horizonte más adecuado para comprender el papel de la universidad y la educación, como tampoco para comprender mejor la contabilidad. (Gómez, 2007, p. 35).

\section{Aspectos generales sobre educación}

Zapata (1997, p. 59) comenta sobre tres alternativas o modelos de diseño curricular universitario: el primero, centrado en el desarrollo del individuo, preferiblemente integrando perfiles de personalidad, habilidades sociales y tendencias en el desarrollo de la disciplina; el segundo modelo, centrado en el objeto de conocimiento de la profesión; y el tercero, centrado en los requerimientos sociales o en cómo se responde a los problemas del contexto. Sin embargo, centrar el currículo en un modelo específico trae muchos riesgos en el proceso educativo, con ventajas y desventajas. Por ejemplo, el primer modelo propuesto es profesionalizante, pragmático y limita el potencial investigativo, pero responde a las demandas del sector productivo; por su parte, el segundo modelo propuesto por el autor potencialmente puede desarrollar conocimiento de punta gracias al desarrollo disciplinario de la propuesta curricular, pero no resuelve los problemas del contexto, y tanto la investigación como la tecnología desarrollada no atenderían las demandas del sector productivo; por último, el tercer modelo puede centrarse en los problemas de la sociedad, pero con un bajo impacto en su resolución al depender científica y tecnológicamente de terceros u otros países. De manera que un modelo curricular de impacto social deberá ser una propuesta que logre integrar los principales elementos de cada modelo enunciado anteriormente.

En general, se percibe la idea de que la educación contable se encuentra en un período de crisis persistente a través del tiempo, lo cual significa que no se ha dado solución a una serie de problemáticas preexistentes, como la relación entre teoría-práctica contable, y surgen nuevas preocupaciones producto de las presiones del contexto, principalmente las relacionadas con la convergencia a normas internacionales de contabilidad y la investigación (Valero y Pinzón, 2013). 
Loaiza (2011, p. 188) propone una revisión bibliométrica de la pedagogía crítica en el marco de la educación contable en Colombia, como referente para repensar la enseñanza de la contabilidad, pero principalmente por las potencialidades "en la comprensión de lo contable".

Una preocupación que se plantea respecto a la formación contable surge con respecto al tipo de educación recibida, lo que Rojas (2008) define como "tecnocrática", que fundamentalmente significa que se basa en lo técnico y deja de lado el sentido crítico. Así mismo, este autor plantea que esto es consecuencia de las motivaciones que tiene el estudiante para ingresar a estudiar Contaduría Pública, por ejemplo, la facilidad por ser una carrera a la que se puede acceder en la jornada nocturna, es decir, se puede trabajar paralelamente, así como el imaginario respecto a un rápido ascenso económico respecto a los profesionales del área. Sobre este tema, Gómez (2012) menciona que una alternativa que están utilizando algunas universidades en Colombia es la problematización de la educación en contaduría, de tal forma que se enfoque no solo en las exigencias legales.

Otro aspecto encontrado en la revisión es el referente a competencias, donde se resalta que su aplicación y concepto "son consecuencia de hechos sociales y económicos presentados en diversos países" (Valero, Patiño y Duque, 2013), de tal forma que, aunque las competencias han sido muy cuestionadas por tendencia internacional, se han ido incorporando como obligatorias por medio de la legislación; Colombia no ha sido la excepción a este fenómeno. Al respecto, Quiroz (2006, p. 84) plantea:

Los estándares proponen unos requerimientos mínimos de calidad, pero el diseño de competencias debe ser un proceso más integral, que recoja las distintas dimensiones de formación, lo cual no va en contravía con la búsqueda de excelencia que debiera caracterizar a los programas académicos.

Lo anterior implica un cuestionamiento acerca de la normativa al respecto, ya que se ha traído de otros lugares sin hacer un análisis detallado de las condiciones del entorno, con las restricciones de la aplicabilidad que esto conlleva. Buqueli, Castillo y Villarreal (2011) plantean la necesidad de 
incentivar el desarrollo de competencias en contabilidad social y dentro de esta el reconocimiento del patrimonio cultural.

Araújo (2000) propone algunos de los cambios que se han dado en la formación en contaduría pública en los últimos treinta años:

Tabla 1. Cambios operados en la contaduría pública en los últimos 30 años

\begin{tabular}{|c|c|}
\hline \multicolumn{2}{|c|}{$\begin{array}{l}\text { Cambios operados en la contaduría } \\
\text { pública (en los últimos } 30 \text { años) }\end{array}$} \\
\hline Ayer & Hoy y mañana \\
\hline 1. Información contable & Conocimiento contable \\
\hline 2. Revisoría fiscal financiera & Revisoría fiscal integral \\
\hline 3. Fe pública & Confianza pública \\
\hline 4. Control interno y control fiscal & Autocontrol \\
\hline 5. Auditoría & Tiende a desaparecer \\
\hline 6. Contabilidad financiera & Además, contabilidad social \\
\hline 7. Técnica contable & Ciencia contable \\
\hline 8. Ética (tradicional) & Una nueva ética \\
\hline $\begin{array}{l}\text { 9. Educación técnica profesional } \\
\text { (basada en el hacer) }\end{array}$ & $\begin{array}{l}\text { Educación formativa:Disciplinal } \\
\text { (basada en el conocimiento) } \\
\text { profesional integral (basada en } \\
\text { la persona) }\end{array}$ \\
\hline
\end{tabular}

Fuente: Tomado de (Araújo, 2000).

En cuanto a los requerimientos sociales respecto a la formación de los contadores públicos, Cuenú (2010), refiriéndose a los estudiantes de Contaduría Pública, plantea: 
El currículo; el cual debe constituirse en la carta de navegación para lograr un sujeto constructor, un estudiante, que se halle siempre inacabado y que esté en la posibilidad de construir conocimiento científico. (Cuenú, 2010, p. 23).

En general, los libros de texto que se han escrito en temas de educación contable se articulan en torno a la reflexión sobre temas diversos. Ya se ha reseñado aquí del trabajo de Cardona y Zapata (2006), en el cual se reúne una amplia serie de reflexiones que hacen exploraciones y análisis alrededor de la historia de la educación contable y un amplio conjunto de temas de relevancia actual (formación, enseñanza, pedagogía y currículo contable), así como la presentación de unos escenarios de prospectiva en los cuales se atienden temas que forman parte de los escenarios de cambio que tensionan el ejercicio educativo y profesional.

La compilación hecha por Gómez y Ospina (2009) de artículos clave del análisis de las corrientes heterodoxas en la disciplina contable a nivel internacional contiene elementos que referencian debates sobre el problema de la educación contable, especialmente en las áreas de la transmisión de ideologías, los poderes en el interior de la academia contable y los desarrollos recientes de los saberes académicos en contabilidad. En general, estos documentos se caracterizan por analizar los problemas de la educación contable desde enfoques no lineales y heterodoxos, que aportan elementos valiosos para la comprensión de las características de la educación contable en Colombia.

La compilación realizada por Rojas (2006) reúne trabajos de análisis y reflexión que incorporan debates en temas de educación contable hechos exclusivamente por autores colombianos. En este libro se destacan dos artículos que introducen reflexiones importantes sobre la educación contable: en primer lugar, el trabajo del mismo Rojas titulado "La paradoja que resulta de la incorporación de las ciencias sociales y humanas en la educación del contador público caso Colombia" (Rojas, 2006), en la cual señala las dinámicas contradictorias que en materia de formación arrojan los contenidos de disciplinas sociales como la sociología, la historia y la 
antropología frente al enfoque estrictamente técnico de la corriente principal de la enseñanza de la contabilidad.

En ese mismo texto está incluido el trabajo de Quintero (2006), en el cual se realizan unas aproximaciones básicas al estado actual de la investigación académica en contabilidad en Colombia, resaltando la debilidad institucional del entorno y la carencia de políticas de promoción de la investigación, así como de grupos consistentes que desarrollen trabajos de largo aliento.

Pero, sin duda, uno de los trabajos de compilación más importantes en el tema de la educación contable en Colombia lo constituye el libro Del hacer al saber: realidades y perspectivas de la educación contable en Colombia, en el cual participan los principales investigadores del Centro Colombiano de Investigación Contable (CCINCO) (Quijano et al., 2002). Este compendio de ensayos presenta un recorrido interesante por los aspectos más polémicos del desarrollo de la educación contable. En general, los documentos remiten a análisis críticos que se refieren a tres elementos: 1) la monotematicidad de la formación contable (negación de la integralidad); 2) el talante técnico, que convierte la formación en instrucción y niega el aporte de la teoría contable; y 3) el bajo nivel de creación de conocimiento, relacionado con una visión pobre sobre el papel de la investigación, la cual se traduce no solo en la formación y la metodología de enseñanza, sino también en los currículos y en las filosofías de los programas.

Otro libro de compendio de ensayos se realizó años más tarde, dirigido en esta oportunidad por Barrios y Rojas (2010), obra en la que se resaltan dos documentos: el de Pesqueux (2010), en el cual se identifica un conjunto de problemáticas relacionadas con la investigación contable, especialmente derivadas del privilegio analítico que se hace a los procedimientos normativos; y el trabajo de Gómez (2010), que hace una descripción muy completa de los desarrollos de la concepción y enseñanza de la teoría contable en Colombia en el período comprendido entre 1970 y 2000, haciendo hincapié en la metodología, las definiciones de teoría contable y, finalmente, las corrientes y escuelas que han dominado el ámbito educativo. 


\section{Conclusiones}

El presente artículo realizó una revisión y análisis de las publicaciones relacionadas con la educación superior contable en el país y evidencia interesantes resultados al respecto. Pero aun a pesar de los avances realizados en términos de cantidad y visibilidad de las publicaciones, se encuentra que los diferentes desarrollos se han dado en el marco de aspectos generales sobre educación; sin embargo, se evidencia que hay categorías que no han sido profundamente trabajadas.

En términos de la clasificación utilizada, es de destacar que, a pesar de contar con nueve categorías, solo dos suman más del $50 \%$ de los textos revisados, siendo estas las de aspectos generales sobre educación y la de metodologías de enseñanza-aprendizaje. Desde esta perspectiva, es clara la tarea pendiente para los investigadores del país en relación con una mayor y profunda investigación en educación contable en Colombia en categorías como la de calidad, historia contable, investigación, productividad académica contable, entre otras.

Es importante resaltar que, aunque existe un incremento de la productividad académica relacionada con el currículo, no en todos los casos se logran socializar las experiencias académicas y en algunos casos los documentos se quedan en aproximaciones netamente teóricas que, si bien son un aporte, no logran evidenciar lo que pasa o podría pasar en la educación superior contable colombiana.

El currículo, como base de la formación de los profesionales del país y como guía en el proceso de enseñanza-aprendizaje, debe ser evaluado constantemente para comprender su construcción frente a los procesos presentes y futuros de la sociedad, sin dejar de lado la importancia de conocer sus desarrollos en el pasado. Siguiendo a Barragán (2009), el currículo de Contaduría debería fortalecerse en el interior de las instituciones de educación superior [IES], de forma que se responda pertinentemente a las necesidades del entorno y no únicamente desde los requerimiento del Estado y el llamado "enfoque tecnocrático", como ha venido sucediendo con 
la categoría "calidad", en el marco de los criterios exigidos por el Consejo Nacional de Acreditación [CNA] y con la investigación desde los referentes de Departamento Administrativo de Ciencia, Tecnología e Innovación [Colciencias].

Frente al plan de estudio como estructura organizativa que contribuye a la gestión de la enseñanza, los resultados giran en torno a que los desarrollos y avances investigativos deben ir encaminados a subsanar o disminuir la segmentación de las asignaturas y la desconexión de los contenidos impartidos, esto por medio de puntos de convergencia o como se viene desarrollando en el Programa de Contaduría de la Universidad de Antioquia, mediante núcleos problémicos que garanticen la integración del contexto, contenidos y procesos de enseñanza-aprendizaje de los diferentes actores involucrados, aspectos que podrían ser potenciados a partir de la socialización de experiencias, de forma que se propicie la formación académica en contexto. En este último aspecto se comienzan a involucrar propuestas que trabajan en la "enseñanza problémica".

Las metodologías de enseñanza-aprendizaje en los documentos revisados evidencian importantes esfuerzos por incrementar la conexión con los estudiantes mediante la aplicación de instrumentos pedagógicos que pueden ir desde lo tradicional a lo alternativo. Entre las diferentes investigaciones es evidente la importancia que tiene el análisis de las TIC en la formación de contadores públicos como un espacio que puede contribuir a disminuir los tiempos en los cuales el conocimiento que se genera adquiera utilidad práctica, por ende, es fundamental el uso de diferentes medios y herramientas (Barreto, Cárdenas y Mondragón, 2011).

Puntualmente, los diferentes procesos de cambio requieren universidades que se transformen en espacios propiciadores de relaciones interdisciplinarias, educadores facilitadores y coautores de su desarrollo, y educandos que estén conscientes de que este tipo de formación les permite evidenciar la necesidad de desarrollar habilidades para comprender y enfrentar la realidad de forma interdisciplinaria. Así, los estudiantes se convierten en un mecanismo de control coincidente dentro de su proceso formativo en 
la medida en que exijan instituciones y docentes con las características de un modelo interdisciplinario (Grajales, 2009).

De la anterior revisión se identifica una preocupación por estudiar el currículo y todos los conceptos que lo componen; especialmente, temas como el plan de estudios y la ética son referentes obligados para varios teóricos al abordar la educación contable. Igualmente, en la revisión bibliográfica se identifica cómo los distintos autores proponen a las instituciones de educación superior una política de pertinencia e impacto social capaz de transformaciones sociales que respondan a las necesidades locales, regionales y nacionales.

Finalmente, se destaca el carácter preponderante que debe tener este tipo de revisiones en Colombia para comprender el punto en el que se encuentra la educación superior contable frente al ejercicio profesional y ante los diferentes requerimientos actuales, como un mecanismo para evidenciar y hacer un llamado de atención en aquellos flancos que se tienen descuidados o poco atendidos. Esta es una forma de alcanzar la tan anhelada "formación integral", que incluya al ser humano como tal, a la sociedad en general, a la cultura y que logre subsanar la eterna disyuntiva disciplinaria de lo técnico y lo teórico, todo ello frente a los retos que impone el contexto y donde las acciones deben ser de carácter propositivo.

\section{Referencias bibliográficas}

Araújo Ensucho, J. A. (2000). Educación superior y contaduría pública. Revista Internacional de Contabilidad \& Auditoría Legis, 2.

Ariza, E. y Soler, E. (2004). La crisis de la universidad. ¿Una lección para reconstruir el tránsito disciplinar de la contabilidad en Colombia? Lúmina, 5, 171-194. Recuperado de goo.gl/aX9hhO

Barragán, D. M. (2009). Curriculum en Contaduría Pública: una visión panorámica de un fenómeno complejo. Trabajo presentado en el I Encuentro Nacional de Profesores de Contaduría Pública. Pontifica Universidad Javeriana, Bogotá, Colombia. 
Barreto-Carvajal, Ó. M., Cárdenas-Mora, S. M. y Mondragón-Hernández, S. A. (2011). Las tecnologías de información y comunicación en la formación de contadores públicos: análisis de uso y aplicaciones en cinco universidades colombianas. Cuadernos de Contabilidad, 12(30), 243-272. Recuperado de goo.gl/ROfr4x

Barrios, C. y Rojas, W. (2010). Perspectivas críticas de la contabilidad contemporánea. Conjunciones y disertaciones: pensando la contabilidad en el siglo XXI. Cali: Universidad del Valle y Pontifica Universidad Javeriana.

Bolívar, A. (2008). Didáctica y curriculum: de la Modernidad a la Postmodernidad. Málaga: Ediciones Aljibe.

Buqueli, M., Castillo, C. y Villareal, J. L. (2011). El patrimonio cultural en los procesos de formación de contadores públicos: un homenaje a la identidad regional. En Memorias del II Encuentro de Profesores de Contaduría Pública. Bogotá D. C.: Universidad Nacional de Colombia y Universidad Santo Tomás.

Cárdenas-Londoño, R. (2009). Papel de las metodologías de enseñanza del proceso lectoescritural en la educación superior. Cuadernos de Contabilidad, 10(26), 87-109. Recuperado de goo.gl/Et1YZz

Cárdenas-Mora, S. M. (2011). Una aproximación al uso de herramientas de gerencia estratégica de costos en instituciones privadas de educación superior. Cuadernos de Contabilidad, 12(31), 547-569. Recuperado de goo.gl/LNHCf6 Cardona, J. (1997). La educación continuada como requisito para el ejercicio profesional en el siglo XXI. Contaduría Universidad de Antioquia, 31, 15-48. Recuperado de goo.gl/Dvmk3V

. (1998). Reflexiones en torno a la relación docencia-investigación en materia contable. Contaduría Universidad de Antioquia, 32, 37-53. Recuperado de goo.gl/qPvjc6

Cardona, J. y Rivera, C. (2002). Acreditación en Contaduría y aseguramiento de la calidad. Contaduría Universidad de Antioquia, 41, 71-112. Recuperado de goo.gl/ncZaWz

Cardona, J. y Zapata, M. (2006). Educación contable: antecedentes, actualidad y prospectiva, Medellín: Universidad de Antioquia.

Celaya-Figueroa, R., López-Parra, M. E. y Aceves-López, J. N. (2008). Ética docente del contador público: un análisis comparativo internacional. Cuadernos de Contabilidad, 9(24), 41-56. 
Cely-Galindo, G. (2011). Educación bioética para vivir, convivir y habitar correctamente. Un plus a la capacitación profesionalizante. Cuadernos de Contabilidad, 12(30), 353-367.

Congreso de Colombia. (13 de julio de 2009). Ley 1314 de 2009. Por la cual se regulan los principios y normas de contabilidad e información financiera y de aseguramiento de información aceptados en Colombia, se señalan las autoridades competentes, el procedimiento para su expedición y se determinan las entidades responsables de vigilar su cumplimiento. Diario Oficial $n .^{\circ} 47.409$ del 13 de julio de 2009. Recuperado de goo.gl/shbqmX

Cubides, H. (1994). Evolución de la capacitación y formación de los contadores públicos. En H. Cubides, A. Maldonado, M. Machado, F. Visbal y É. Gracia, Historia de la contaduría pública en Colombia. Siglo XX (pp. 35-133). Bogotá: Universidad Central. ¿QUÉ EDICIÓN ES DE 1994?

Cuenú, J. (2010). Impedimientos de los estudiantes de Contaduría Pública para formarse como sujetos constructores de conocimiento científico. Revista Internacional de Contabilidad \& Auditoría Legis, 44, 119-138.

Díaz, A. (2006). La enseñanza problémica o problematizadora. Una adecuada estrategia pedagógica para mejorar las competencias cognitivas en la educación contable. Lúmina, 7, 98-109. Recuperado de goo.gl/OmDK6K

Giraldo, X. (2013). Contabilidad y diversidad cultural: apuntes para pensar un proyecto educativo en contaduría. En Memorias III Encuentro Nacional de Profesores de Contaduría Pública. Desarrollos y desafíos de la educación contable en Colombia. Cali: Pontificia Universidad Javeriana y Universidad del Valle.

Gómez, M. (2007). Las insuficiencias disciplinares de los estándares internacionales de educación -IES- para contadores profesionales. Lúmina, 8, 24-42.

. (2010). Dinámica de la concepción y la enseñanza de la teoría contable en Colombia (1970-2000). En C. Barrios y W. Rojas (Comps.), Perspectivas críticas de la contabilidad contemporánea. Conjunciones y disertaciones: pensando la contabilidad en el siglo XXI (pp. 127-160). Cali: Universidad del Valle y Universidad Javeriana.

Gómez, M. y Ospina, C. (2009). Avances interdiscipinarios para una comprensión crítica de la contabilidad, Medellín: Universidad de Antioquia y Universidad Nacional de Colombia. 
Gómez, Y. (2012). Educabilidad: una resignificación conceptual en contabilidad desde la educación crítica. Contaduría Universidad de Antioquia, 61, 91-110. Recuperado de goo.gl/NzOcve

Gonzalo Angulo, J. A. y Garvey, A. M. (2009). Ética y enseñanza de la contabilidad (una propuesta de discusión). Contaduría Universidad de Antioquia, 50, 11-42. Recuperado de goo.gl/VFXJBw

Gracia, E. (2011). La investigación contable en el acto educativo. En Memorias II Encuentro de profesores de Contaduría Pública. Bogotá D.C.: Universidad Nacional de Colombia y Universidad Santo Tomás.

Grajales-Sánchez, P. A. (2009). Producción intelectual e interdisciplinariedad. Cuadernos de Contabilidad, 10(27), 205-208. Recuperado de goo.gl/PnMgLW

Herrera, F. (2013). Los dilemas morales como estrategia pedagógica para la formación ética del contador público. En Memorias III Encuentro Nacional de Profesores de Contaduría Pública. Desarrollos y desafíos de la educación contable en Colombia. Cali: Pontificia Universidad Javeriana y Universidad del Valle.

León-Paime, E. F. (2011). Ser y comprender al docente contable: exploraciones autoetnográficas. Cuadernos de Contabilidad, 12(30), 179-210. Recuperado de goo.gl/7FIwtK

Loaiza, F. (2011). Producción académica sobre educación contable en Colombia 2000-2009: incidencia de la pedagogía crítica. Lúmina, 12, 172-194. Recuperado de goo.gl/LNQiYl

Martínez, A. (2011). Una doble calzada: la técnica contable y la didáctica creativa. Nueva lógica de posibilidades pensada desde las sensaciones hasta las razones. En Memorias II Encuentro de Profesores de Contaduría Pública. Bogotá D.C.: Universidad Nacional de Colombia y Universidad Santo Tomás.

Moreno, M. (2006). El trabajo grupal sistémico como estrategia de aprendizaje en los espacios académicos del contador público. Lúmina, 7, 52-63.

Ospina Zapata, C. M. (2009). Educación contable en Colombia. Sentires de algunos actores y la educación contable como acción educativa. Trabajo presentado en el I Encuentro Nacional de Profesores de Contaduría Pública. Pontificia Universidad Javeriana, Bogotá, Colombia.

Patiño-Jacinto, R. A. y Santos, G. (2009). Plan de estudios de Contaduría Pública en Colombia y propuestas de formación profesional. Revista Internacional de Contabilidad \& Auditoría Legis, 37, 131-164. 
Pesqueux, I. (2010). Los problemas actuales en la investigación en contabilidad y en control: el problema de la norma. En C. Barrios y W. Rojas (Comps.), Perspectivas críticas de la contabilidad contemporánea. Conjunciones y disertaciones: pensando la contabilidad en el siglo XXI (pp. 61-84). Cali: Universidad del Valle y Pontifica Universidad Javeriana.

Quijano, O. et al. (2002). Del hacer al saber: realidades y perspectivas de la educación contable en Colombia. Popayán: Universidad del Cauca.

Quintero, H. (2006). Aproximaciones a la investigación contable. En J. A. SuárezPineda (Comp.), La contabilidad en la Modernidad: referentes filosóficos y social-históricos (pp. 81-103). Cali: Universidad del Valle.

Quiroz, E. H. (2006). Competencias formativas y mercado profesional contable. Revista Internacional de Contabilidad \& Auditoría Legis, 26, 143-196.

Revelo, L. y Bennett, A. (2013). Análisis de las necesidades del mercado laboral de San Juan de Pasto frente a la propuesta formativa del Programa de Contaduría Pública de la Universidad Mariana. En Memorias III Encuentro Nacional de Profesores de Contaduría Pública. Desarrollos y desafíos de la educación contable en Colombia. Cali: Pontificia Universidad Javeriana y Universidad del Valle.

Ríos, R. A. (2013). La formación para la investigación de contadores públicos. Revista Internacional de Contabilidad \& Auditoría Legis, 54, 77-142.

Rodríguez, F. B. (2008). Las redes empresariales y la dinámica de la empresa: aproximación teórica. INNOVAR. Revista en Ciencias Administrativas y Sociales, 18, 27-47. Recuperado de goo.gl/SbeFTG

Rodríguez-Castro, B. (2010). Retos de la academia contable. Cuadernos de Contabilidad, 11(28), 11-13. Recuperado de goo.gl/X89Q79

Rojas, W. (2006). La contabilidad en la Modernidad: referentes filosóficos y socialhistóricos. Cali: Universidad del Valle.

. (2008). Congoja por una educacón contable fútil. Contaduría Universidad de Antioquia, 52, 259-274. Recuperado de goo.gl/eJ4JpR

Rojas, W. y Giraldo, G. (2013). Humanidades y educación contable: categoría indivisible para pensar la formación del contador público. En Memorias III Encuentro Nacional de Profesores de Contaduría Pública. Desarrollos y desafíos de la educación contable en Colombia. Cali: Pontificia Universidad Javeriana y Universidad del Valle.

Roncancio, A. y Camargo, D. (2013). El texto inalterable, una mirada a la educación tradicional en la enseñanza de la contabilidad. En Memorias III Encuentro 
Nacional de Profesores de Contaduría Pública. Desarrollos y desafíos de la educación contable en Colombia. Cali: Pontificia Universidad Javeriana y Universidad del Valle.

Rueda-Delgado, G (2013). La enseñabilidad de lo internacional y la globalización en la contabilidad: ¿la única respuesta en la convergencia? En Memorias III Encuentro Nacional de Profesores de Contaduría Pública. Desarrollos y desafíos de la educación contable en Colombia. Cali: Pontificia Universidad Javeriana y Universidad del Valle.

(2011). El papel de las publicaciones científicas para la docencia. Cuadernos de Contabilidad, 12(31), 427-430.

. (2009a). Orígenes de la producción académica contable. Cuadernos de Contabilidad, 10(26), 9-11. Recuerado de goo.gl/lgd2Qm

. (2009b). Enfoque y estructura curricular. Bogotá: Pontificia Universidad Javeriana.

Sánchez, W. (2011). Valores contables: competencias socioafectivas para contaduría pública. Armenia: Investigar Editores.

Santana, P. J. (2009). ¿Es la gestión de calidad total en educación un nuevo modelo organizativo? HEURESIS. Revista Electrónica de Investigación Curricular y Educativa, 26(6), 1-15. Recuperado de goo.gl/gQGRdl

Valderrama-Prieto, M. A., Castro-Rodríguez, B., Arias-Bello, M. L., Bejarano, D. F., Garzón, H. A., Salgado-Castillo, J. A., Rueda-Delgado, G., Espinosa-Flórez, G. E. y Sosa-Cardozo, J. M. (2009). El plan de estudios del programa de Contaduría Pública de la Pontificia Universidad Javeriana frente a los retos que genera la Ley 1314 de 2009. Cuadernos de Contabilidad, 10(26), 113-145. Recuperado de goo.gl/br1Q8k

Valero, G. y Patiño-Jacinto, R. A. (2010). Aproximación a la educación contable a través de la revisión de journals internacionales. En L. Y. Pérez-Meneses e I. Y. Ramos-Moreno, El rol de la teoría y la investigación contable en el desarrollo de la sociedad (pp. 1-24). Santa Marta: Universidad Cooperativa de Colombia. Valero, G., Patiño-Jacinto, R. A. y Duque, O. (2013). Competencias para el programa de Contaduría Pública: una aproximación conceptual. Contaduría Universidad de Antioquia, 62, 11-36. Recuperado de goo.gl/kIV1c7

Valero, G. y Pinzón, J. (2013). Las miradas a la formación de contables: percepciones de los estudiantes acerca de los problemas en los programas de Contaduría 
Pública. En Memorias III Encuentro Nacional de Profesores de Contaduría Pública. Desarrollos y desafíos de la educación contable en Colombia. Cali: Pontificia Universidad Javeriana y Universidad del Valle.

Vásquez Bernal, R. (2002). Nuevos desafíos de la educación frente a estándares internacionales de formación en contaduría pública. Cuadernos de Contabilidad, 19, 151-156. Recuperado de goo.gl/Bzr95k

Villareal, J. L. (2011). De la formación conceptual al aprendizaje significativo de la auditoría. En Memorias II Encuentro de profesores de Contaduría Pública. Bogotá D.C.: Universidad Nacional de Colombia y Universidad Santo Tomás. Zapata, M. (1998). Paradojas en las propuestas de rediseño curricular. Lúmina, $2,71-87$.

Zapata Monsalve, M. Á. (1997b). Paradojas en las propuestas de rediseño curricular. Trabajo presentado en el II Congreso Interamericano de profesores del área contable. Lima, Perú.

Zubiri, X. (1987). Naturaleza, historia, dios, Madrid: Editorial Alianza. 


\section{ANEXOS}

Tabla 2. Composición de documentos revisados por fuente

\begin{tabular}{l|c|c}
\hline Fuente & $\begin{array}{c}\text { Documentos } \\
\text { revisados }\end{array}$ & Porcentaje \\
\hline Libros & 18 & $16 \%$ \\
\hline Revista Contaduría Universidad de Antioquia & 11 & $10 \%$ \\
\hline Revista Cuadernos de Contabilidad & 18 & $16 \%$ \\
\hline $\begin{array}{l}\text { Revista Internacional LEGIS de Contabilidad } \\
\text { Auditoría }\end{array}$ & 6 & $5 \%$ \\
\hline Revista Lúmina & 6 & $5 \%$ \\
\hline $\begin{array}{l}\text { Memorias I Encuentro Nacional de Profesores } \\
\text { de Contaduría Pública }\end{array}$ & 43 & $38 \%$ \\
\hline $\begin{array}{l}\text { Memorias II Encuentro Nacional de Profesores } \\
\text { de Contaduría Pública }\end{array}$ & 5 & $4 \%$ \\
\hline $\begin{array}{l}\text { Memorias III Encuentro Nacional de } \\
\text { Profesores de Contaduría Pública }\end{array}$ & 7 & $100 \%$ \\
\hline \begin{tabular}{l} 
Total \\
\hline
\end{tabular}
\end{tabular}

Fuente: elaboración propia. 
96 ACTivos | Ruth Alejandra Patiño Jacinto, Gloria Milena Valero Zapata, Martha

Giovanna Acosta Sahamuel, José Fabián Parra Acosta, Jairo Alonso Bautista

\begin{tabular}{|c|c|c|c|c|c|c|c|c|c|c|c|}
\hline$\partial^{0}$ & $\begin{array}{l}\circ \\
\text { वे }\end{array}$ & $\stackrel{\circ}{m}$ & $\stackrel{\circ}{\forall}$ & $\stackrel{\circ}{n}$ & $\begin{array}{l}a^{0} \\
\infty\end{array}$ & $\stackrel{\circ}{\sim}$ & $\begin{array}{l}0 \\
m\end{array}$ & $\begin{array}{l}\stackrel{\circ}{\infty} \\
\stackrel{\infty}{-1}\end{array}$ & $\begin{array}{l}\therefore \\
m \\
=\end{array}$ & $\stackrel{+}{+}$ & $\begin{array}{l}\circ \\
8\end{array}$ \\
\hline 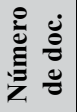 & $\stackrel{2}{7}$ & $m$ & in & $\infty$ & $a$ & $\sim$ & $m$ & 이 & $\stackrel{2}{2}$ & 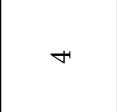 & $\stackrel{\Xi}{\Xi}$ \\
\hline$\stackrel{m}{\stackrel{\sim}{\sim}}$ & $m$ & & - & & - & & - & - & $m$ & & 으 \\
\hline సี่ & $\sim$ & & & & & & & - & & & $m$ \\
\hline$\overline{\bar{~}}$ & $m$ & & & $\neg$ & $\sim$ & & & $m$ & - & $\sim$ & $\simeq$ \\
\hline 록 & $\sim$ & & & - & & & & & & & $m$ \\
\hline હે & $\approx$ & & $n$ & in & $m$ & - & & 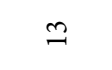 & $\curvearrowright$ & $\sim$ & L \\
\hline$\stackrel{\infty}{\stackrel{\overbrace{}}{े}}$ & $\sim$ & - & & & - & & & & & & 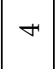 \\
\hline ڤ્స & & - & & & & & & & & & - \\
\hline ๕్̀ે & $\theta$ & & & & & & & $\sim$ & & & 0 \\
\hline ఫ્స & & & & & & & & & -1 & & - \\
\hline ฮิ & in & - & & & & & & & - & & $\wedge$ \\
\hline ఫ్ & - & & & & & & - & & & & $\sim$ \\
\hline$\stackrel{2}{\circ}$ & & & & & & & & & - & & - \\
\hline$\hat{\sigma}$ & - & & - & - & - & & - & & - & & 0 \\
\hline ฮั & & & & & & - & & & & & - \\
\hline$\stackrel{\hat{0}}{\sigma}$ & & & & & - & & & & & & - \\
\hline 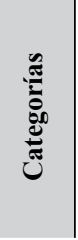 & 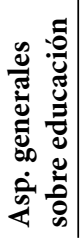 & ن & 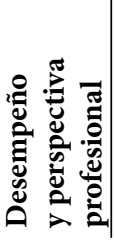 & 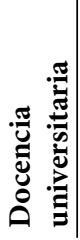 & : & 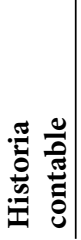 & 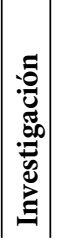 & 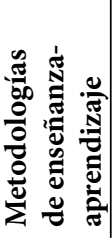 & 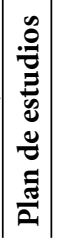 & 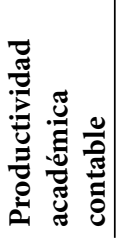 & 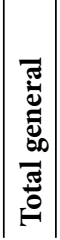 \\
\hline
\end{tabular}

Universidad Santo Tomás, Facultad de Contaduría Pública 
Figura 1. Participación por género

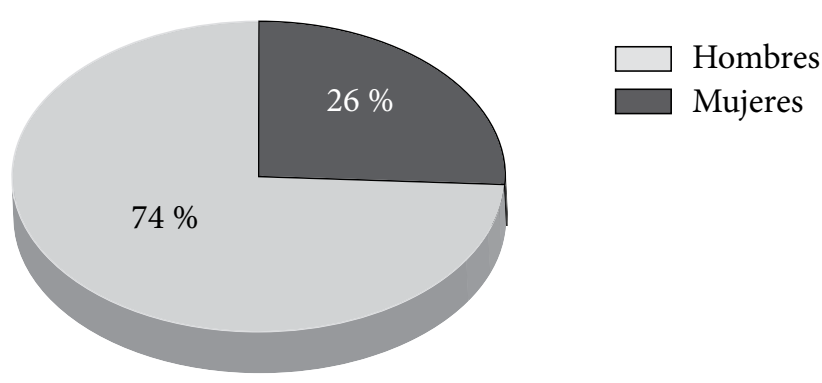

Fuente: elaboración propia. 
98 ACTivos | Ruth Alejandra Patiño Jacinto, Gloria Milena Valero Zapata, Martha

Giovanna Acosta Sahamuel, José Fabián Parra Acosta, Jairo Alonso Bautista

\begin{tabular}{|c|c|c|c|c|c|c|c|c|c|c|c|}
\hline$\partial^{0}$ & $\stackrel{\circ}{\circ}$ & $\stackrel{\circ}{m}$ & $\stackrel{\circ}{m}$ & $\stackrel{\circ}{m}$ & $\stackrel{\circ}{m}$ & $\stackrel{\circ}{\sim}$ & $\stackrel{\circ}{\sim}$ & $\stackrel{\leftrightarrow}{\sim}$ & $\stackrel{\circ}{\sim}$ & $\stackrel{\circ}{\sim}$ & $\left|\begin{array}{l}0 \\
0 \\
0\end{array}\right|$ \\
\hline 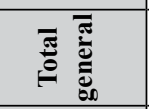 & 6 & in & in & $r$ & $r$ & $m$ & $m$ & $m$ & $m$ & $m$ & ले \\
\hline 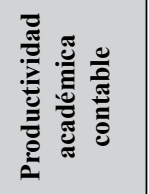 & & & N & & & & & & & & N \\
\hline 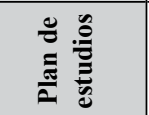 & $\sim$ & & $\sim$ & & - & & $\neg$ & - & & & $\wedge$ \\
\hline 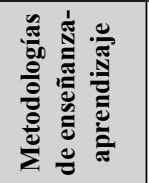 & & & & & & & & & & - & - \\
\hline 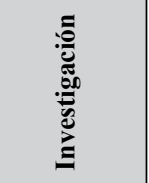 & - & & & & & & & & & & - \\
\hline 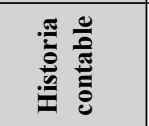 & & & & & & & & & & & 0 \\
\hline 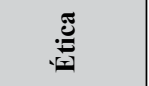 & & $\neg$ & & & & & & & & & - \\
\hline 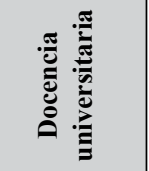 & & $\neg$ & & $\neg$ & & & & & & - & $n$ \\
\hline 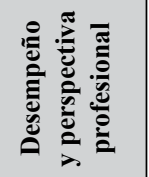 & $\neg$ & & & $\neg$ & & & & & & & 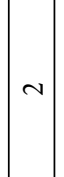 \\
\hline Uू & & $\neg$ & & - & & - & & & & & $m$ \\
\hline 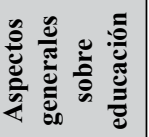 & $\sim$ & $\sim$ & - & $\neg$ & $m$ & $\sim$ & $\sim$ & $\sim$ & $m$ & $\neg$ & 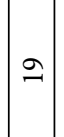 \\
\hline 总 & 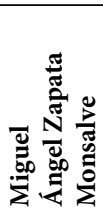 & 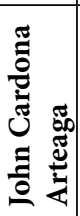 & 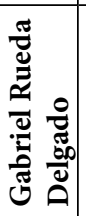 & 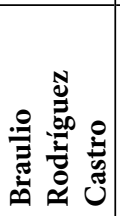 & 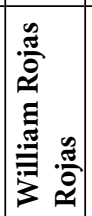 & 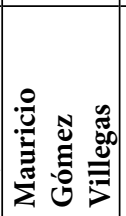 & 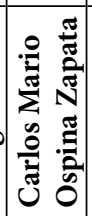 & 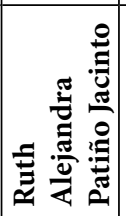 & 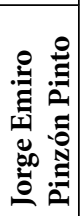 & 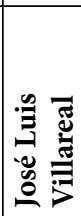 & 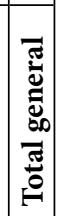 \\
\hline
\end{tabular}




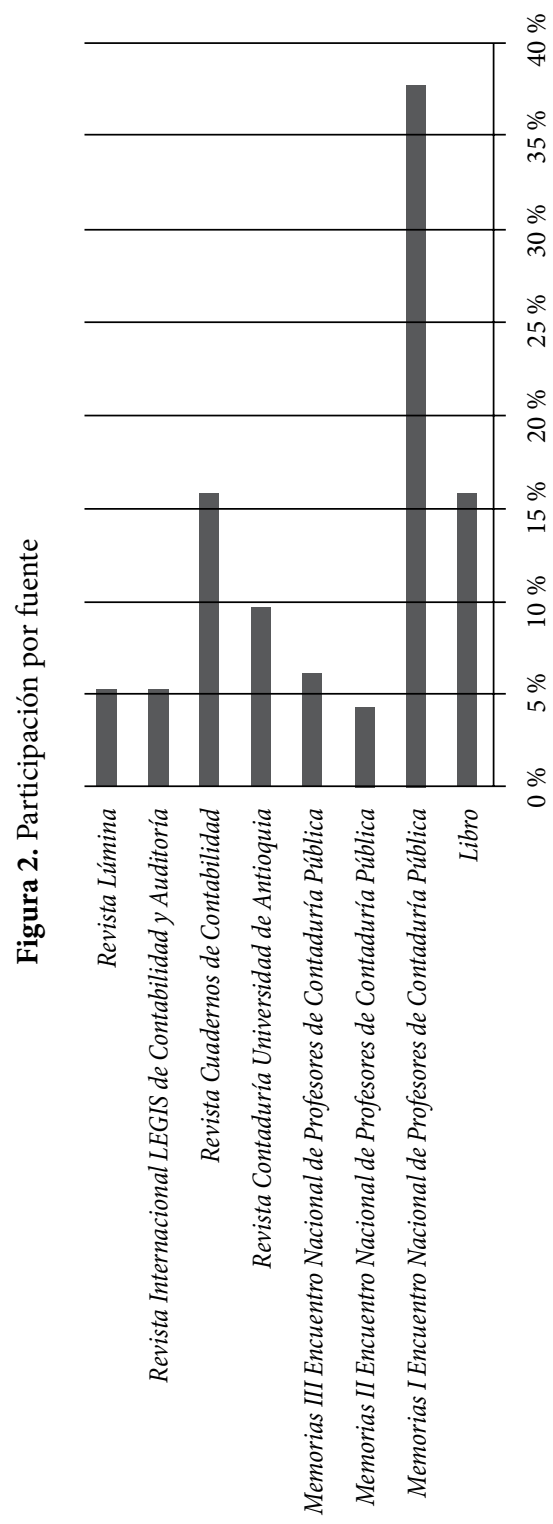

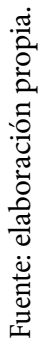

ISSN: 0124-5805, Revista Activos, N. ${ }^{\circ} 26$, enero-junio de 2016, pp. 61-99 